\title{
Toward an Agile System: Iranian Information System for Covid-19-Affected Patients Data Collection from Iranian Hospitals
}

\author{
Somayeh ABEDIAN ${ }^{\mathrm{a}, \mathrm{b}, 1}$, Pirhossein KOLIVAND ${ }^{\mathrm{a}}$, Hamid Reza LORNEJAD ${ }^{\mathrm{a}}$ \\ a National Emergency Medical Services Organization, Ministry of Health and Medical \\ Education, Tehran, I.R. Iran \\ b Department of Information Technology Management, Islamic Azad University, \\ Qazvin, Iran
}

\begin{abstract}
COVID-19's rapid spreads has caused a global pandemic. On $19^{\text {th }}$ February 2020, Iran reported its first confirmed cases of infections in Qom City and the number of diagnosed cases and the death toll rose exponentially in March [1-3]. Managing the disease, which is considered a pandemic according to the World Health Organization (WHO) [4], requires definite approaches differing according to various factors in each country, which may also lead to (in)effective dealing with the disease. In addition, using international data and information, and WHO advice, especially in the crisis and therapeutic procedures, is one of the best crisis management strategies [5]. For every plan by governances, the first step is collecting information on epidemic distribution for the purpose of isolating provinces and cities at a national scale. Thus, Ministry of Health and Medical Education of Iran (MOHME) attempted to collect the minimum required data on the infection-affected patients based on medical records and epidemiological factors, such as demographic data (gender, age and national code), exposure history (close contact with the infected, suspect patients or even having traveled) and signs and symptoms (fever, cough, shortness or difficulties in breathing, fatigue, anorexia, hemoptysis, sputum production, dyspnea, Myalgia, Pharyngalgia, nausea, vomiting, Diarrhea, Headache, Abdominal pain, Dizziness, etc.). Therefore, to ensure accuracy and validity, and to speed up data collection in an area, Information Technology (IT) tools were required [6]. In this regard, developing an information system with a simple format and userfriendly interface in the shortest possible time was the aim. This study presents the local information system developed in March 2020, which has been registering hospitalized Covide-19-affected patients in Iranian hospitals up till now. In other words, this paper introduces features and procedures of one of the national systems as a health registry that includes clinical information on admitted Covid-19 patients in Iranian hospitals from admission to discharge or death. This system is supported by MOHME, and along with outpatient Point of Care Information Systems (POCS), feeds the national and international pandemic reports and decisions.
\end{abstract}

Keywords. COVID-19; crisis management; information technology, Health Information System (HIS), Case Report Form (CRF)

${ }^{1}$ Corresponding Author: Somayeh Abedian, Ministry of Health and Medical Education, Tehran, Iran, Email: s.abedian@behdasht.gov.ir, so.abedian@gmail.com 


\section{Introduction}

In February 2020, some countries were already fighting COVID-19, and after China, the Islamic Republic of Iran was the second country to declare two deaths due to coronavirus, within 50 days after China on February $18^{\text {th }}, 2020$. It is surmised that the virus may have been transmitted to the country by a merchant from Qom who had travelled to China [1].

During the COVID-19 outbreak, more than in any other public health conditions, Health Information Systems (HIS) perform a critical role in information management [7]. The policies are made according to crucial evidence by health professionals in taking the post appropriate action, making the most informed decisions to allow for better insights on measures with the aim of improving citizens' healthcare. Emerging automation and information have the potential to improve public health. HISs arrange for immediate, expeditious, and coordinated data access and sharing, and they facilitate the care priority and access, especially for the people in vulnerable conditions. With properly disaggregated data, it is possible to plan activities that reduce probable health inequities at all levels of care, and facilitate the implementation of strategies to address such cases of injustice. In addition, they provide reliable statistical information for planning and healthcare with respect to human resources allocation, and medicine and medical equipment assignment [8-10].

After the first case of the outbreak was detected in Iran, COVID-19 Disease Management Policy Committee (CDMPC) was informed at the MOHME. The committee approved to collect information of the patients suffering pneumonia with acute respiratory distress from hospitals as the main healthcare providing centers with cases of severe illness. According to the experts' opinion and solutions analysis, in seeking an Agile HIS, the use of a national web-based system was considered.

This paper presents the experience of Iran in applying a live and dynamic system for data gathering about the infection-affected (or suspicious of Covide-19) patients from hospitals. The process would include reports from the data entry at the hospitals to monitoring dashboard development for medical science universities and MOHME.

\section{Methods}

With COVID-19 outbreak, countries need to adopt a broad and long-term vision to improve data systems to help manage information about the pandemic. A wholegovernment, multi-sectoral approach is required to use the outbreak data to manage consumer supplies, hospital beds, sharing of resources among healthcare provider centers and deployment of healthcare workers [11]. This article aims to introduce the live system that is utilized in Iran for data gathering from hospitals. It focuses on the patients referred to a hospital, suspected of an acute respiratory condition. The system's role is similar to a health registry for decision making, while facing the effects of COVID-19, in terms of prevention and control, based on the collected and processed data, as well as the received and generated information. This national project benefited from the professional advice of public health professionals, IT experts and CDMPC members. Based on committee statements in the limited time for crisis management, use or development of a national integrated system require some phases and steps throughout the process as following: 


\subsection{Data Requirements and Modeling}

Information systems have played a crucial role in reacting to the outbreak of COVID-19, not only they have served as platforms for efficient and effective communications but they have also essentially changed the dynamics of interactions among healthcare providers and patients, and the healthcare services delivery methods [12-13]. This should be based on commonly agreed-upon standardized data and fit-for-purpose indicators in the use of HISs infrastructures. In this project, the primary phase was developing optimal clinical and non-clinical data elements identification and Case Report Form (CRF) design since there was not any template announced by WHO at the start of the pandemic. Thus, CDMPC members discussed the main goals of CFR count and related items, which led to three finalized CFR categories.

1. Hospitalized pneumonia report form containing admission and demographics (date-time of admission, date of initial symptoms prior to diagnosis, full name, nationality, national code, age, gender, weight, travel history, level of education, occupation status, etc.), Past Medical History (PMH: pre-existing health conditions such as diseases or pregnancy, smoking, addiction or substance abuse, medications, etc.), clinical services, examinations (signs and symptoms), any type of Covid-19 test results (such as PCR test, lg test and CT scan), reinfection information, etc.

2. Discharge report form containing date of discharge, conditions on discharge, prescribed medical orders, etc.

3. Death report form containing time of death, infection intensity (Mild, Moderate, Severe or Critical), Covide-19 virus identification or suspicion, and cause of death based on ICD10.

Indeed, after CRF's first version was published to be confirmed by WHO, it was revised [14].

\subsection{Software Development Process}

In the first step, using hospital information systems were recommended, but this idea was rejected because of the variety in the types of products (more than 30 software programs with different technology) and challenges in their data integration. The second reachable solution was discussed around the utilization of national Electronic Health Record (EHR). Iranian EHR receives data from all POCSs based on WSDL standards and essential components of SOA architecture [15], but the challenge of time of data transferring from hospitals still persisted since the data were provided after patients' discharge (inpatient clinical items) and MOHME needs them as early as the pandemic is picking up. Therefore, this solution was rejected too (for this goal, development of new online web services was both necessary and time-consuming and saving time was very important to us at the beginning of the pandemic).

Finally, according to experts' experience, using a national web-based system developed earlier in order to manage patient transferring process among hospitals was selected due to the following reasons:

1. The system's technical infrastructure was ready and development and launch time would be saved.

2. The system was form-based and designing new forms was possible.

3. Hospital users were healthcare providers already familiar with the system (more than 1200 hospitals). They are trained to work with the system. 
4. Usernames and passwords are defined for users.

5. There are supervisory units in the universities of medical sciences to monitor the accuracy of data entry in electronic forms, which assures MOHME of evidence accuracy and validity.

6. The system was integrated on a national scale and did not require software or new web-service development.

According to above-mentioned points, CRFs were developed in the system and MOHME required the hospitals to fill them twice a day (12 a.m. and 2 p.m.).

\subsection{Supervision and Revision}

After one week, the results of CFRs fillings were evaluated. Based on the results of supervisory report on more than 1200 hospitals (governmental, private, charity, military and other), CDMPC members provided some recommendations, and consequently the system was updated for the purpose of better data analysis.

\subsection{Regional/National Dashboard Development}

MOHME has developed one multi-resource monitoring dashboard. It shows current and reliable data on COVID-19 cases submitted directly from healthcare provider centers and is used by decision makers at regional and national access level. In addition, the dashboard has a clean and modern interface with several data visualization tools to better grasp the current status of COVID-19 as the situation unfolds [16]. The dashboard resources were supplied by HISs (such as this project for hospital cases, laboratory information systems, primary care information systems, mobile-based or home-care systems and clinical information systems) including information on the hospitalized/outpatient definite or suspected patients based on WHO guidelines. To this aim, MOHME released a number of substantial indicators to visualize and monitor COVID-19 outbreak dispersion seeking the following issues:

1. New and confirmed cases, recovered and deaths nationally with daily statistics.

2. City and province-specific information by clicking on any city/province/medical science university on the interactive map with intelligent alarms such as color.

3. Interactive charts showing the reported cases by regions including daily and cumulative statistics and weekly, monthly and annual trends.

4. Confirmed cases, deaths, and changes over time in a specific province, region, or territory, on interactive charts.

5. A new explorer tab designed to provide complex datasets for easy access and use. It allows health policy-makers to select variables which help spot correlations and relationships that can provide insights into COVID-19 and how communities are responding to it.

It is critically important that all healthcare centers specially hospitals be able to register their COVID-19 situations. The powered dashboard will now provide more comprehensive insights into the epidemiology and response to COVID-19 at regional and national scales. 


\section{Results}

Health information systems are one of the six WHO health system building blocks: "A well-functioning health information system is one that ensures the production, analysis, dissemination and use of reliable and timely information on health determinants, health system performance and health status" [6]. With the COVID-19 outbreak, the most basic issue was the spreading virus volume in the country which was manageable just by means of an electronic information system to meet reliable and timely evidence.

In this paper, an agile information system development for COVID-19 outbreak monitoring in Iranian hospitals is introduced. It depicts a multi-step process from planning, CRF design and case registration to monitoring dashboard development which is shown in Figure 1.

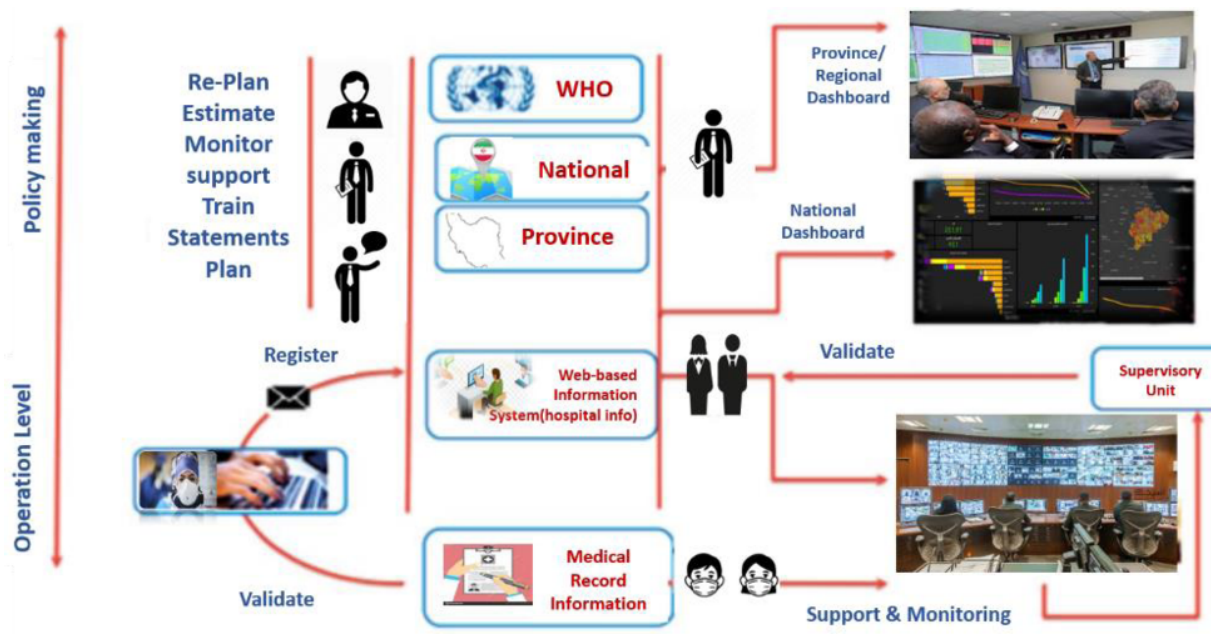

Figure 1. The COVID-19 data collection process from Iranian hospitals

\section{Discussion}

A national information system is designed based on professional advice and emerging requirements. In this regard, a committee consisting of all relevant experts was formed as early as the first coronavirus case declaration in Iran. In this process, supported by the Iranian Ministry of Health and Medical Education, attempts were made to improve the reliability and validity of COVID-19-affected patients' information and statistics.

This paper seeks to illustrate a nation-wide effort to provide and manage valid information on the COVID-19 pandemic for public health planning in the country. The government is using this data repository beside other clinical data resources in order to improve the quality of health services acceptability, continuity/sustainability, clinical effectiveness in a minimum time and prevent further spreading of the virus. Moreover, national reports shown in the dashboard feed the national and international reports. 


\section{Acknowledgment}

The authors express their gratitude to Dr. Saeid Namaki (Minister of Health and Medical Education) for his supports and all efforts throughout COVID-19 pandemic in Iran. We would also like to appreciate our experts for their advices especially Dr. Mohammad Hussein Norouzi.

\section{References}

[1] York Times. "How Iran Became a New Epicenter of the Coronavirus Outbreak". New Archived from the original on 27 June 2021. Available from: https://www.newyorker.com/news/our-columnists/how-iranbecame-a-new-epicenter-of-the-coronavirus-outbreak

[2] Iranian Ministry of Health and Medical Education. Coronavirus epidemic in Iran. Available from: http://ird.behdasht.gov.ir/. Accessed August 3, 2020.

[3] Venkatesan P. COVID-19 in Iran: round 2. Lancet Infect Dis. 2020 Jul;20(7):784. doi: 10.1016/S14733099(20)30500-4. PMID: 32592672; PMCID: PMC7314444.

[4] Prevention UCfDCa. Coronavirus Disease 2019 (Covid-19). (2020). Available online at: https://www.cdc.gov/coronavirus/2019-nCoV/summary.html

[5] Rassouli Maryam, Ashrafizadeh Hadis, Shirinabadi Farahani Azam, Akbari Mohammad Esmaeil, "COVID-19 Management in Iran as One of the Most Affected Countries in the World: Advantages and Weaknesses", JOURNAL: "Frontiers in Public Health", VOLUME:8, 2020, Available from: https://www.frontiersin.org/article/10.3389/fpubh.2020.00510, DOI: 10.3389/fpubh.2020.00510, ISSN:2296-2565

[6] Al-Shorbaji N. E-health in the Eastern Mediterranean Region: a decade of challenges and achievements. East Mediterr Health J. 2008;14 Suppl: S157-73. PMID: 19205616.

[7] Pan American Health Organization," COVID-19 and the importance of strengthening Information Systems, PAHO/EIH/IS/COVID-19/20-0021, 2020-05-12, Available at: https://iris.paho.org/handle/10665.2/52127

[8] WHO Regional Office for Africa 2020, Monitoring and evaluation framework for the Covid-19 response in the WHO African Region. Available https://apps.who.int/iris/bitstream/handle/10665/334344/9789290234432-eng.pdf

[9] World Health Organization. Everybody's business: strengthening health systems to improve health outcomes: WHO's framework for action. Production [Internet]. 2007;1-56. Available from: http://www.who.int/healthsystems/strategy/everybodys business.pdf

[10] Saxena N, Gupta P, Raman R, Rathore AS. Role of data science in managing COVID-19 pandemic. Indian Chemical Engineer, 2020; 62(4): 385-395. DOI: 10.1080/00194506.2020.1855085 To link to this article: https://doi.org/10.1080/00194506.2020.1855085

[11] Ros F, Kush R, Friedman C, Gil Zorzo E, Rivero Corte P, Rubin JC, et al. Addressing the Covid-19 pandemic and future public health challenges through global collaboration and a data-driven systems approach. Learn Health Syst. 2020 Dec 6;5(1):e10253. doi: 10.1002/lrh2.10253

[12] Mykytyn PP. COVID-19 and Its Impacts on Managing Information Systems, Information Systems Management. 2020;37(4):267-271. DOI: 10.1080/10580530.2020.1818900

[13] Duckett S. What should primary care look like after the COVID-19 pandemic? Australian Journal of Primary Health 2020;26(3):207-211. https://doi.org/10.1071/PY20095

[14] World Health Organization. Revised case report form for confirmed novel coronavirus COVID-19 (report to WHO within 48 hours of case identification), 27 February 2020. Available from: https://apps.who.int/iris/handle/10665/331234?show=full

[15] Abedian S, Kazemi H, Riazi H, Bitaraf E. Cross hospital bed management system. Stud Health Technol Inform. 2014; 205:126-130. PMID: 25160159.

[16] Irwansyah E, Budiharto W, Widhyatmoko D, Istamar A, Putra Panghurian F. Monitoring Coronavirus COVID-19/SARS-CoV-2 Pandemic using GIS Dashboard: International and Indonesia Context. Preprints 2020, 2020080415 (doi: 10.20944/preprints202008. 0415.v1). 